\title{
PTEN induces apoptosis and cavitation via HIF-2-dependent Bnip3 upregulation during epithelial lumen formation
}

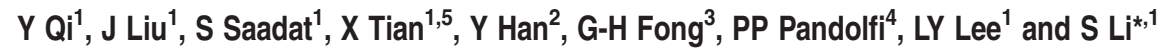

The tumor suppressor phosphatase and tensin homolog (PTEN) dephosphorylates PIP3 and antagonizes the prosurvival PI3K-Akt pathway. Targeted deletion of PTEN in mice led to early embryonic lethality. To elucidate its role in embryonic epithelial morphogenesis and the underlying mechanisms, we used embryonic stem cell-derived embryoid body (EB), an epithelial cyst structurally similar to the periimplantation embryo. PTEN is upregulated during EB morphogenesis in parallel with apoptosis of core cells, which mediates EB cavitation. Genetic ablation of PTEN causes Akt overactivation, apoptosis resistance and cavitation blockade. However, rescue experiments using mutant PTEN and pharmacological inhibition of Akt suggest that the phosphatase activity of PTEN and Akt are not involved in apoptosis-mediated cavitation. Instead, hypoxia-induced upregulation of Bnip3, a proapoptotic BH3-only protein, mediates PTEN-dependent apoptosis and cavitation. PTEN inactivation inhibits hypoxia- and reactive oxygen species-induced Bnip3 elevation. Overexpression of Bnip3 in PTEN-null EBs rescues apoptosis of the core cells. Mechanistically, suppression of Bnip3 following PTEN loss is likely due to reduction of hypoxia-inducible factor-2 $\alpha$ (HIF-2 $\alpha$ ) because forced expression of an oxygen-stable HIF-2 $\alpha$ mutant rescues Bnip3 expression and apoptosis. Lastly, we show that HIF-2 $\alpha$ is upregulated by PTEN at both transcriptional and posttranscriptional levels. Ablation of prolyl hydroxylase domain-containing protein 2 (PHD2) in normal EBs or inhibition of PHD activities in PTEN-null EBs stabilizes HIF-2 $\alpha$ and induces Bnip3 and caspase-3 activation. Altogether, these results suggest that PTEN is required for apoptosis-mediated cavitation during epithelial morphogenesis by regulating the expression of HIF-2 $\alpha$ and Bnip3.

Cell Death and Differentiation (2015) 22, 875-884; doi:10.1038/cdd.2014.185; published online 14 November 2014

\section{Introduction}

The formation of epithelial tissues is fundamental to both development and normal physiology, whereas its deregulation can lead to cancer. During periimplantation embryogenesis, the pluripotent inner cell mass of the blastocyst is converted from a nonpolar cell aggregate to a highly organized epithelial cyst. This morphogenetic transformation involves the formation of a polarized epiblast epithelium along with apoptosismediated removal of the core cells. ${ }^{1}$ Despite the fundamental importance of these processes, both for embryonic development and epithelial biology, there is limited understanding of the underlying molecular mechanisms.

Phosphatase and tensin homolog (PTEN) is an important tumor suppressor and its inactivation causes tumors of breast, prostate and many other organs. ${ }^{2}$ An initial study of its physiological functions in vivo demonstrated that targeted deletion of PTEN in C57BL/6 J mice caused embryonic death before E7.5. ${ }^{3}$ This was confirmed shortly thereafter by Podsypanina et al. ${ }^{4}$ who observed disorganized PTEN-null embryos at E6.5 undergoing a process of resorption. Another study showed that the mutant mice die at E9.5 with patterning defects in the CD1/C57BL genetic background. ${ }^{5}$ The reason for this discrepancy in the stage of embryonic lethality is unknown. Nonetheless, these results indicate that PTEN is required for embryogenesis, likely germ layer formation.

In Drosophila, loss of PTEN in the developing wing inhibits junction lengthening and cell rearrangement, thereby disrupting epithelial planar polarity. ${ }^{6}$ In addition, PTEN is essential for polarized assembly of the apical membrane of rhabdomeres. ${ }^{7}$ In cell culture experiments, shRNA/siRNA-mediated reduction of PTEN in Madin-Darby canine kidney and mammary epithelial cells prevents the formation of the apical surface and polarized epithelial architecture. ${ }^{8,9}$ These results suggest that PTEN is implicated in epithelial tissue formation.

PTEN functions as both a lipid and a dual-specificity protein phosphatase. Its well-documented lipid phosphatase activity dephosphorylates $\mathrm{PIP}_{3}$, thereby antagonizing the prosurvival PI3K-Akt pathway. ${ }^{10}$ Biochemical analyses have identified several phosphoproteins including focal adhesion kinase as

\footnotetext{
${ }^{1}$ Department of Surgery, Rutgers University Robert Wood Johnson Medical School, 125 Paterson Street, New Brunswick, NJ 08093, USA; ${ }^{2}$ Shenyang Northern Hospital, Shenyang, China; ${ }^{3}$ Center for Vascular Biology, University of Connecticut Health Center, Farmington, CT 06030, USA and ${ }^{4}$ Beth Israel Deaconess Cancer Center, Harvard Medical School, Boston, MA 02215, USA

*Corresponding author: S Li, Department of Surgery, Rutgers-Robert Wood Johnson Medical School, 125 Paterson Street, New Brunswick, NJ 08903, USA. Tel: +1 732 235 6164; Fax: +1 732235 6003; E-mail: shaohua.li@ rutgers.edu

${ }^{5}$ Current address: Shenyang Northern Hospital, Shenyang, China.

Abbreviations: PTEN, phosphatase and tensin homolog; PIP3, phosphatidylinositol (3,4,5)-trisphosphate; PI3K, phosphoinositide 3-kinase; EB, embryoid body; Bnip3, BCL2/adenovirus E1B $19 \mathrm{kDa}$ protein-interacting protein 3; HIF-2 $\alpha$, hypoxia-inducible factor-2 $\alpha$; PHD2, prolyl hydroxylase domain-containing protein 2; shRNA/siRNA, short hairpin RNA/small interfering RNA; ROS, reactive oxygen species; MUPP1, multi-PDZ domain protein 1; PDZ, post synaptic density protein 95, disc large, and zonula occludens-1; FoxO3a, forkhead box O3a; AIF, apoptosis-inducing factor; Bax, Bcl-2-associated X protein; Bak, Bcl-2-antagonist/killer Received 17.2.14; revised 23.9.14; accepted 01.10.14; Edited by M Piacentini; published online 14.11.14
} 
substrates of the PTEN protein phosphatase activity. ${ }^{11,12}$ There is also increasing evidence of important phosphataseindependent activities of PTEN that contribute to its tumor suppressor functions. ${ }^{13-15}$ However, the relative contributions of the lipid and protein phosphatase activities, as well as phosphatase-independent functions, to the roles of PTEN in epithelial morphogenesis are unknown.

We use embryonic stem (ES) cell-derived embryoid bodies (EBs) to study embryonic epithelial morphogenesis. ${ }^{16,17}$ When cultured in suspension, ES cell aggregates undergo compaction and form spherical EBs. The outer cells of the EB differentiate to become endoderm, which deposits an underlying basement membrane. The epiblast cells adjacent to the basement membrane polarize to become a columnar epithelium, whereas the remaining core cells not in contact with the basement membrane die by caspase-dependent-apoptosis (Supplementary Figure S1), creating a proamniotic-like cavity. ${ }^{18,19}$ These differentiation processes recapitulate periimplantation development and provide a tractable in vitro model for the study of epithelial polarization and apoptosismediated cavitation. In this study, we demonstrate that PTEN is essential for embryonic epithelial morphogenesis. By reconstituting PTEN-null EBs with wild-type and mutant PTEN, we show that PTEN promotes apoptosis and cavitation independently of its phosphatase activity and Akt. We further show that PTEN is required for hypoxia- and reactive oxygen species (ROS)-induced upregulation of Bnip3, a BH3-only proapoptotic protein, which induces the apoptosis of the core cells and cavitation. These results demonstrate a new mechanism whereby PTEN promotes apoptosis and lumen formation during epithelial morphogenesis.

\section{Results}

Ablation of PTEN inhibits apoptosis and cavitation. To investigate the role of PTEN in embryonic epithelial morphogenesis, we analyzed the differentiation of ES cell-derived EBs. During EB cavitation, PTEN was upregulated at both protein and mRNA levels in parallel with reduced Akt phosphorylation and increased caspase-3 activation (Figures 1a and d). Immunofluorescence demonstrated that PTEN was highly expressed in the core cells in 3-day EBs before cavitation and was enriched on both the basal and apical sides of the polarized epiblast in 5-day EBs (Figure 1b). Similarly, PTEN was present in the centrally located cells in E6.0 mouse embryos where apoptosis is detected (Figure 1c). ${ }^{19}$ Compared with wild-type controls, PTEN - / - EBs formed endoderm and the basement membrane, but displayed markedly reduced caspase- 3 activation in the core cells (Figures 1e and f). As a consequence, the mutant EBs failed to cavitate. In addition, the epiblast cells in contact with the basement membrane could not polarize as evidenced by lacking an apical actin belt and absent apical accumulation of the polarity marker MUPP1 (Figure 1f). These results suggest that PTEN is required for apoptosis-mediated cavitation and epiblast polarization.

The phosphatase domain of PTEN is dispensable for apoptosis induction. PTEN consists of an N-terminal phosphatase domain and a C-terminal domain. The mutation of glycine 129 to glutamic acid (G129E) in the phosphatase domain selectively abolishes its lipid phosphatase activity, whereas the $\mathrm{C} 124 \mathrm{~S}$ mutation inactivates both lipid and protein phosphatase activities. ${ }^{20,21}$ The C-terminal domain contains a membrane-interacting $\mathrm{C} 2$ domain and a PDZ-binding motif. To investigate the structure-function relationship of PTEN in embryonic epithelial morphogenesis, we reconstituted PTEN - / - EBs with wild-type and mutant PTEN by stable transfection (Figures 2a-d). Rescue of PTEN-/ - EBs with wild-type PTEN suppressed Akt phosphorylation and completely restored epiblast polarization and apoptosis-mediated cavitation, whereas transfection with the vector alone had no effect (Figures 2b and e-h). Unexpectedly, the G129E mutant, which failed to suppress Akt overactivation, also rescued apoptosis and cavitation, as well as apical polarization of the epiblast as revealed by the formation of an apical actin belt and localization of MUPP1 and syntaxin-3 to the apical surface (Figure 2e and data not shown). However, the epiblast height was only half of normal controls or wild-type PTEN-rescued mutant EBs (Figures $2 e$ and $h$ ). These results indicate that the lipid phosphatase activity of PTEN is dispensable for apoptosis-mediated cavitation and apical polarization but is required for epiblast elongation. By contrast, reconstitution of PTEN - / - EBs with the C124S mutant failed to rescue apical polarity or cavitation, although central apoptosis developed normally (Figures $2 \mathrm{e}$ and $\mathrm{g}$ ). This suggests that the protein phosphatase activity of PTEN is required for epiblast polarization and clearance of apoptotic cells in the EB center. The PTEN mutant that lacks the $\mathrm{N}$-terminal phosphatase domain (PTEN $\triangle \mathrm{N}$ ) could also restore apoptosis of the core cells, whereas the $\mathrm{C} 2$ domain transfection failed to do so (Figure 2e). Altogether, these results demonstrate a division of labor among the lipid and protein phosphatase activities, as well as phosphatase-independent functions of PTEN in the regulation of epithelial elongation, apical polarization and apoptosis-mediated cavitation.

\section{Apoptosis resistance and cavitation blockade following} PTEN ablation is not caused by Akt overactivation. In the absence of PTEN, PIP3 accumulates at the plasma membrane and recruits phosphoinositide-dependent kinase 1 (PDK1) and Akt. PDK1 phosphorylates Akt at threonine 308 in the activation loop and mammalian target of rapamycin complex 2 phosphorylates serine 473 at the $C$ terminus, and consequently leads to full activation of Akt. We observed a significant increase in Akt phosphorylation at these two sites in PTEN-/- EBs, suggesting overactivation of Akt (Figure 3a). To determine if Akt overactivation might contribute to the apoptosis resistance after PTEN ablation, we treated PTEN - / - EBs for 4 days with Akt1/2 inhibitor, which reduced Akt phosphorylation at both $\mathrm{S} 473$ and T308 but was unable to induce apoptosis and cavitation (Figures $3 \mathrm{~b}$ and $\mathrm{c}$ ). To further assess the role of Akt activation, we stably overexpressed constitutively active, myristoylated Akt1 in normal EBs (Figure 3d). Compared with the GFP control, overexpression of myristoylated Akt increased phosphorylation of Akt and its substrate but did not alter the level of caspase-3 activation in 3-day EBs (Figure 3d). These results suggest that the resistance of the core cells to apoptosis in PTEN - / - EBs is unlikely caused by Akt overactivation. 
a

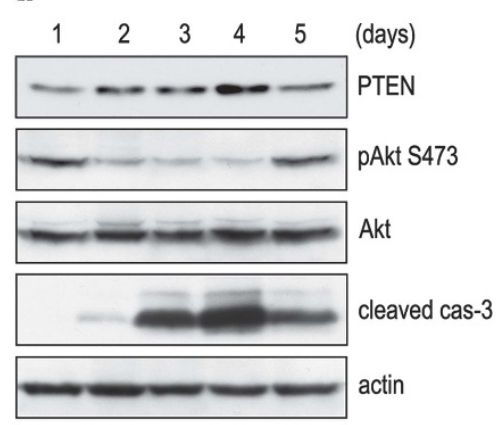

d

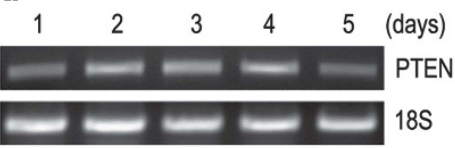

e

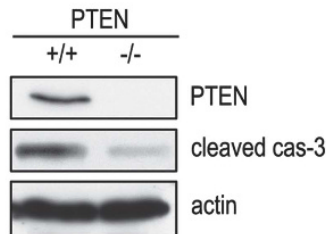

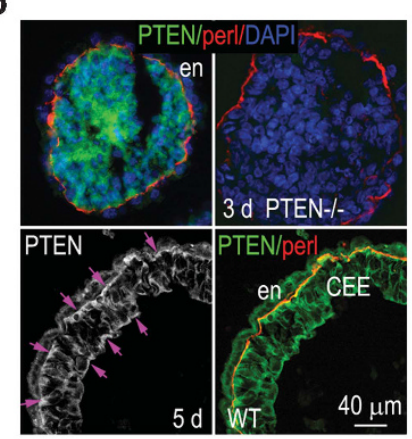

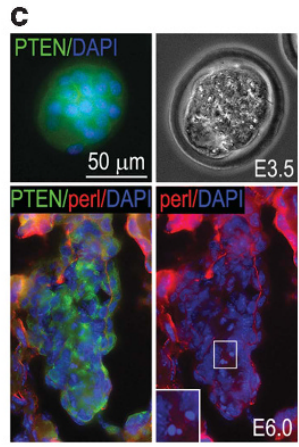

f

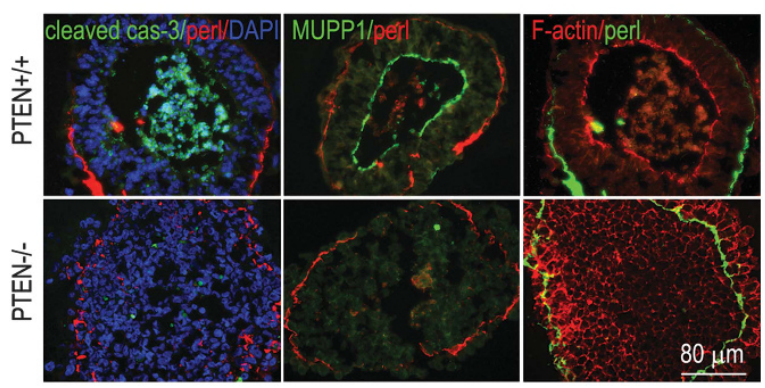

Figure 1 Ablation of PTEN inhibits apoptosis, cavitation and epiblast polarization. (a) Normal EBs cultured for 1-5 days were analyzed by immunoblotting for PTEN, phosphoAkt Ser 473 (pAkt S473), Akt and cleaved caspase-3 (cas-3). Actin serves as a loading control. Expression of PTEN was increased in 2-, 3- and 4-day EBs, in parallel with reduced Akt phosphorylation and increased cas-3 activation. (b) Immunostaining showed that PTEN was highly expressed in the centrally located cells in 3-day EBs and was redistributed to the apical and the basal side of the epiblast epithelium in 5-day EBs (arrows). PTEN - / - EBs serve as a negative control for PTEN immunostaining. (c) E3.5 (early blastocyst stage) and E6.0 embryos were immunostained for PTEN and perlecan. PTEN was enriched in the centrally located cells. (d) Reverse transcription-PCR (RT-PCR) showed that PTEN mRNA levels were increased in 2-, 3- and 4-day EBs. (e) Three-day PTEN+/+ and PTEN - / - EBs were analyzed by immunoblotting for PTEN and cleaved cas-3. Ablation of PTEN inhibited cas-3 activation. (f) Five-day EBs were immunostained for cleaved cas-3 and the apical polarity marker MUPP1. Basement membrane was identified by perlecan immunofluorescence. F-actin was stained with rhodamine-phalloidin. Nuclei were counterstained with DAPI (4',6-diamidino-2-phenylindole). Ablation of PTEN inhibited apoptosis of the core cells and blocked cavitation. The epiblast cells of the mutant EBs failed to form a polarized apical domain

We also evaluated the contribution of the forkhead transcription factor FoxO3a, a major downstream effector of Akt involved in apoptosis induction and ROS metabolism, to cell death during EB cavitation. ${ }^{22}$ Akt phosphorylates FoxO3a at T32, S253 and S315, causes its retention in the cytoplasm and consequently antagonizes its apoptosis-inducing activity. ${ }^{23}$ Cytoplasmic and nuclear fractionation showed no reduction of nuclear FoxO3a, which is unphosphorylated and transcriptionally active ${ }^{23}$ in PTEN - / - EBs despite increased cytoplasmic levels (Supplementary Figure S2A). Furthermore, forced expression of either the active form (T32A/S253A/S315A, the mutant lacks the three Akt phosphorylation sites for nuclear export) or the inactive form (H212R, the mutant lacks the transcriptional ability because of its inability to bind to DNA) of FoxO3a had no significant effect on casplase-3 activation compared with wild-type FoxO3a-transfected EBs (Supplementary Figure S2B). These results suggest that FoxO3a does not contribute to apoptosis of the core cells.

PTEN induces apoptosis of the core cells through Bnip3. Recently, we have shown that Bnip3 is upregulated in the core cells and cooperates with apoptosis-inducing factor (AIF) to induce apoptosis and cavitation during EB morphogenesis. ${ }^{19}$ To determine whether AIF and Bnip3 may be implicated in PTEN-dependent apoptosis, we first examined the expression of AIF and Bnip3 in PTEN+/+ and
PTEN - / - EBs. As shown in Figure 4a, the expression of AIF was largely unchanged at the protein level in the absence of PTEN. By contrast, Bnip3 upregulation was significantly inhibited in PTEN - / - EBs, which correlated with reduced caspase-3 activation (Figure 4b). As Bnip3 is induced in the core cells by hypoxia, we tested whether PTEN is required for hypoxia-induced Bnip3 expression. ${ }^{19}$ We incubated 1-day PTEN+/+ and PTEN - / - EBs in hypoxia pouches for $16 \mathrm{~h}$ and then analyzed Bnip3 expression by immunoblotting. In PTEN+/+ EBs, hypoxia induced a robust increase of Bnip3; conversely, Bnip3 expression was completely abolished in PTEN-/- EBs (Figure 4c), suggesting that PTEN is required for hypoxia-induced Bnip3 expression. We then surmised that if the reduced Bnip3 expression in PTEN - / EBs is responsible for the resistance of the core cells to apoptosis, forced expression of Bnip3 should restore apoptosis. Indeed, stable expression of Bnip3 in 2-day PTEN - / - EBs markedly increased caspase-3 activation compared with PTEN+/+ and PTEN -/ - EBs expressing GFP alone (Figure 4d). Immunostaining for cleaved caspase-3 confirmed apoptosis occurring mostly in the core cells (Figure 4e). Massive apoptosis was observed in the EBs without basement membranes, which spread to the outer layer and led to the disintegration of EBs in 3 to 4 days. Despite enhanced apoptosis, cavitation did not occur even in EBs cultured for up to 10 days. These results suggest 
a

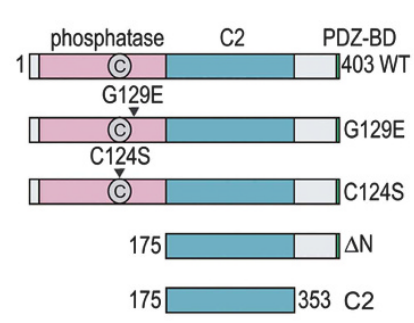

b

PTEN+/+ PTEN-I-

GFP GFP WT G129E C124S

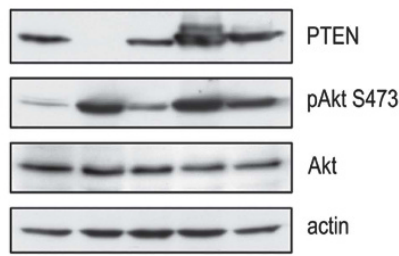

c

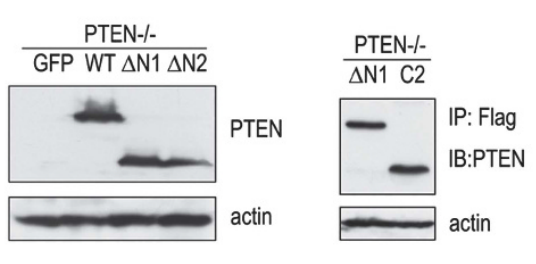

e $\quad$ PTEN+/+ GFP GFP

PTEN-/-

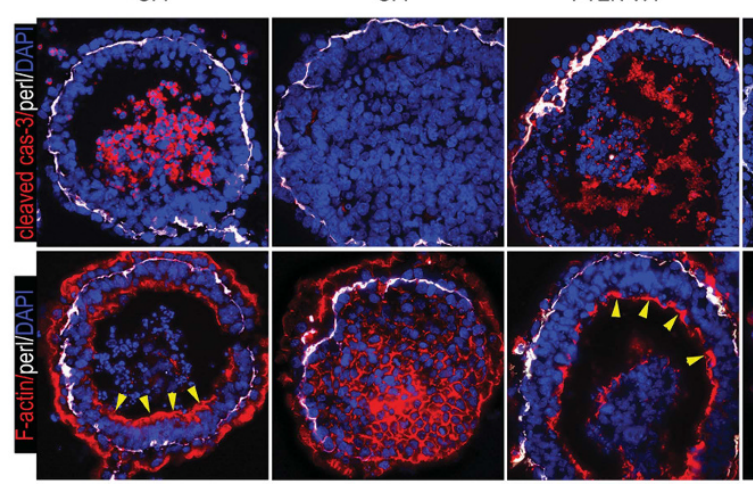
PTEN-G129E
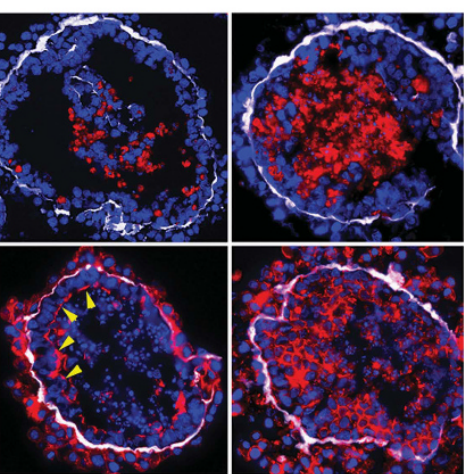

PTEN-AN PTEN-C2

f

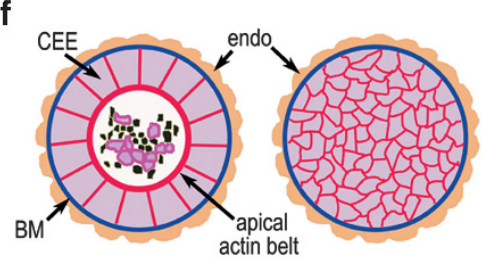

g

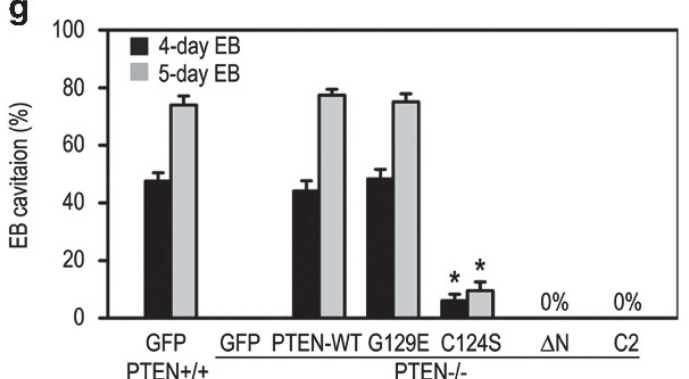

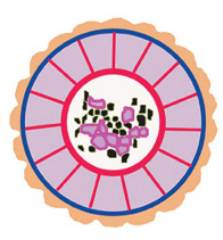

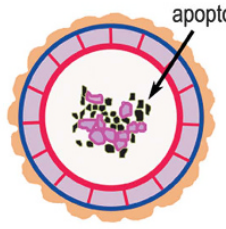

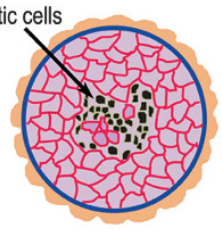
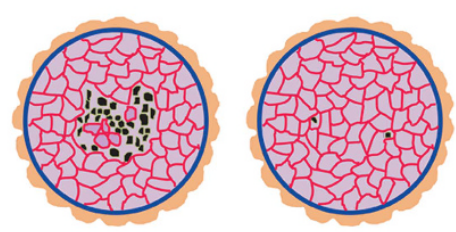

h

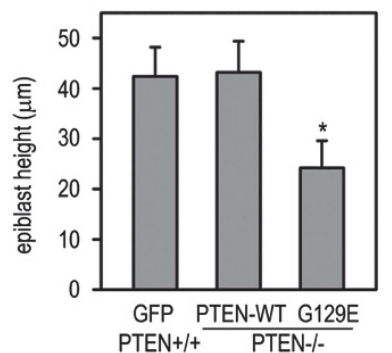

Figure 2 Structure-function analysis of PTEN in embryonic epithelial morphogenesis. (a) Diagrams show PTEN mutagenesis. (b) PTEN+/+ EBs expressing green fluorescent protein (GFP) and PTEN - / - EBs expressing GFP, wild-type (WT) PTEN, PTEN-G129E or PTEN-C124S were cultured for 4 days. Immunoblotting confirmed the expression of WTand mutant PTEN in PTEN - / - EBs. Reconstitution of PTEN - / - EBs with WT PTEN suppressed Akt phosphorylation, whereas transfection with G129E or C124S had no effect. Total Akt was largely unchanged in all these clones. (c) Immunoblot analysis confirmed the expression of PTEN $\Delta N$ in 4-day PTEN - / - EBs. (d) Four-day EBs were immunoprecipitated with anti-Flag Ab followed by immunoblotting with anti-PTEN Ab. Actin was used as a loading control. The expression level of the C2 domain was comparable to PTEN $\Delta \mathrm{N}$ clone 1 ( $\Delta \mathrm{N} 1$ ), which was used in the subsequent analysis. (e) Five-day EBs were immunostained for cleaved caspase-3 (cas-3) and perlecan. F-actin was stained with rhodamine-phalloidin. Nuclei were counterstained with DAPI (4',6-diamidino-2-phenylindole). Arrowheads indicate the apical actin belt. (f) Diagrams of EB polarization, apoptosis and cavitation corresponding to immunofluorescence images in panel e. (g) EBs with a central cavity were counted by live-phase microscopy and plotted as a percentage of total EBs examined ( $n=7$ independent experiments with a total of $894-1131$ EBs for each group). C124S versus PTEN $+/+$ GFP and PTEN $-/-$ rescued with PTEN-WT ( $\left.{ }^{*} P<0.01\right)$. (h) Epiblast height was measured in 5 -day EB sections ( $n=50$ for each group). PTEN $-/-+$ G129E versus PTEN $+/+$ GFP and PTEN $-/-+$ PTEN-WT $\left({ }^{\star} P<0.01\right)$. BM, basement membrane; $\mathrm{CEE}$, columnar epiblast epithelium; endo, endoderm

that Bnip3 is upregulated during EB morphogenesis in a PTEN-dependent manner and mediates apoptosis of the core cells.

An elegant study using mouse embryonic fibroblasts deficient in Bax and Bak showed that Bnip3 induces apoptosis via activation of these two proapoptotic proteins. ${ }^{24}$ To explore the role of Bax and Bak in Bnip3-mediated apoptosis during EB cavitation, we performed immunoblotting on Bnip3knockdown EBs using antibodies (Abs) that recognize the active form of Bak (Bak NT) and Bax (the 6A7 epitope). 
a

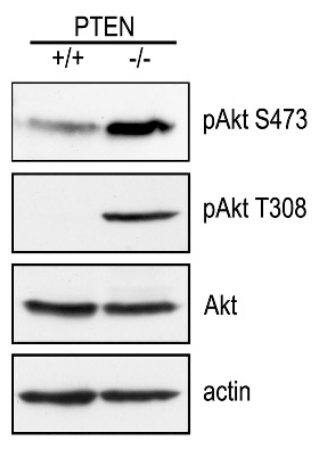

c

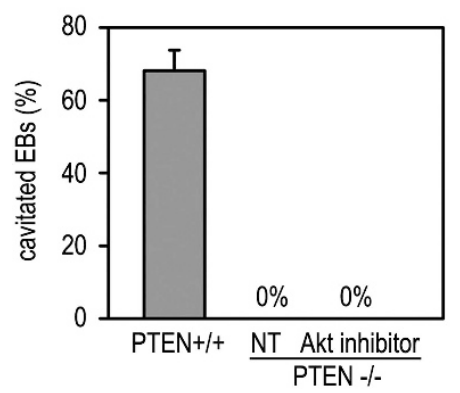

b

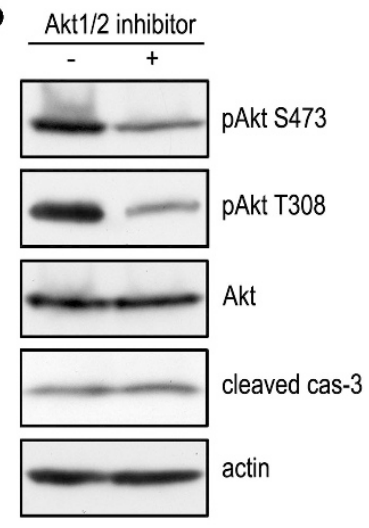

d

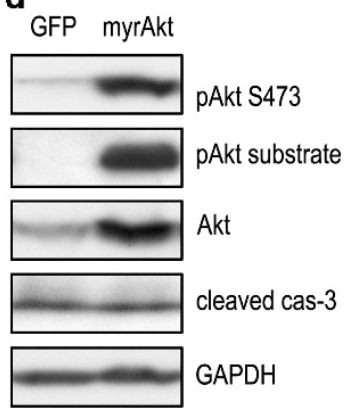

Figure 3 The inhibition of apoptosis and cavitation following PTEN ablation is not caused by Akt overactivation. (a) Immunoblot analysis showed that ablation of PTEN in 3-day EBs significantly increased phosphorylation of Akt at both S473 and T308, whereas total Akt remained largely unchanged. (b) One-day PTEN - / - EBs were cultured in the presence or absence of $150 \mathrm{nM} \mathrm{Akt1/2}$ inhibitor for additional 4 days and subjected to immunoblot analysis. Treatment of Akt1/2 inhibitor reduced Akt phosphorylation at both S473 and T308 but did not increase caspase-3 (cas-3) activation. (c) EBs with a central cavity were counted by live-phase microscopy and plotted as a percentage of total EBs examined ( $n=5$ independent experiments with a total of 933-1071 EBs for each group). None of PTEN - / - EBs, either untreated (NT) or treated with Akt $1 / 2$ inhibitor, cavitated. (d) Normal ES cells were stably transfected with constitutively active, myristoylated Akt1 (myrAkt) or green fluorescent protein (GFP) alone. Overexpression of myrAkt increased the level of phospho-Akt S473 (pAkt) and phospho-Akt substrate but had no effect on cas-3 activation in 3-day EBs. GAPDH, glyceraldehyde 3-phosphate dehydrogenase

We observed no significant change in Bak and Bax activation in Bnip3-depleted EBs (Supplementary Figure S3C). In addition, ablation of PTEN did not influence Bak NT and the Bax 6A7 epitope either. Unlike Bnip3, which is localized to the hypoxic core cells (Supplementary Figure S3D), ${ }^{19}$ both Bak NT and Bax (6A7) were detected in all the cells in 3-day EBs and colocalized with the mitochondria marker complex $\mathrm{V}$ (Supplementary Figure S3D and data not shown). However, immunoblot analysis under non-reducing conditions showed no obvious oligomer formation of both Bak and Bax in control and Bnip3-knockdown EBs (data not shown). These results suggest that Bak and Bax are unlikely to mediate Bnip3dependent apoptosis and cavitation.

The $\mathbf{C}$ terminus of PTEN is required for Bnip3 upregulation during cavitation. To determine the PTEN domains involved in Bnip3 upregulation, we cultured PTEN+/+ GFP EBs and PTEN - / - EBs stably transfected with GFP, wildtype PTEN or various mutants for 2 and 4 days. Immunoblot

analysis showed that Bnip3 was expressed at low levels in 2-day PTEN+/+ GFP EBs and was markedly induced after 4 days when cavitation was in rapid progression (Figure 5). However, the upregulation of Bnip3 was abrogated in PTEN - / - GFP EBs. Reconstitution of the mutant EBs with wild-type PTEN, G129E, C124S or PTEN $\triangle \mathrm{N}$ all restored Bnip3 expression, whereas transfection with the $\mathrm{C} 2$ domain only had no effect. The expression level of Bnip3 was in parallel with that of caspase-3 activation. These results indicate that the $\mathrm{C}$ terminus of PTEN is required for Bnip3 upregulation and apoptosis induction during EB cavitation.

PTEN regulates Bnip3 expression and apoptosis downstream of ROS through HIF-2a. We have previously shown that Bnip3 upregulation during EB cavitation is mediated by AIF-dependent ROS production in mitochondria and the induction of hypoxia-inducible factor-2a (HIF-2a), which is selectively stabilized in the hypoxic EB center. ${ }^{19}$ To determine whether PTEN regulates ROS production, we stained 3-day EBs with the fluorescent indicator dihydroethidium (DHE), which emits red fluorescence after being oxidized by ROS. DHE fluorescence was mainly observed in the core cells in both PTEN+/+ and PTEN - / - EBs and was not significantly changed in the absence of PTEN (Figure 6a). We next examined whether PTEN is required for ROS-induced Bnip3 expression and apoptosis by incubating 1-day EBs with $0.1 \mathrm{mM} \mathrm{H}_{2} \mathrm{O}_{2}$ for $24 \mathrm{~h} . \mathrm{H}_{2} \mathrm{O}_{2}$ treatment increased Bnip3 and cleaved caspase-3 in PTEN+/+ but not PTEN-/- EBs (Figure 6b), suggesting that PTEN is downstream of ROS. To test if PTEN regulates Bnip3 expression through HIF-2a, we analyzed HIF-2a expression by immunoblotting. Although the HIF-2a protein was readily detectable in 4-day PTEN+/+ GFP EBs, its level was much lower in PTEN-/- GFP EBs (Figure 6c). Reconstitution of PTEN - / - EBs with wild-type, G129E or C124S PTEN all restored the HIF-2a protein to near normal levels. This result suggests that PTEN maintains the HIF-2a protein level in a phosphatase-independent manner. When 1-day EBs were cultured in a hypoxia pouch for $16 \mathrm{~h}, \mathrm{HIF}-2 a$ was significantly increased in PTEN+/+ but not PTEN - / - EBs (Figure 6d). To test if HIF-2a mediates PTEN-dependent Bnip3 elevation and apoptosis, we stably expressed in PTEN - / - ES cells constitutively active mutant HIF-2a PPN (P405A, P531A, N847Q) that is stable under normoxia but still binds to DNA and activate transcription, as described previously (Figure 6e). ${ }^{19}$ PTEN - / - EBs expressing GFP or HIF-2a PPN were cultured for 3 days and analyzed for Bnip3 and cleaved caspase-3 by immunoblotting. The expression of HIF-2 $a$ PPN resulted in a substantial elevation of Bnip3 and cleaved caspase-3 (Figure 6f). Altogether, these results suggest that PTEN induces Bnip3 expression and apoptosis downstream of ROS through HIF-2a.

HIF-2 $a$ is regulated by PTEN both transcriptionally and posttranscriptionally. To determine whether PTEN regulates HIF-2a transcription, we performed RT-PCR on 4-day EBs. Densitometric quantitation revealed that the mRNA for HIF-2 $a$ was reduced by $\sim 50 \%$ after PTEN ablation, suggesting that reduced HIF-2a transcription may contribute to the decreased protein levels (Figures $7 a$ and b). In normoxia, the 
a

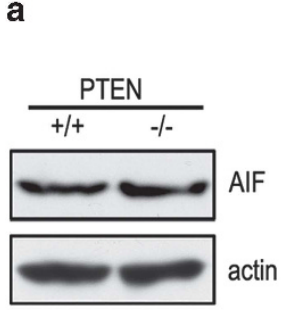

b

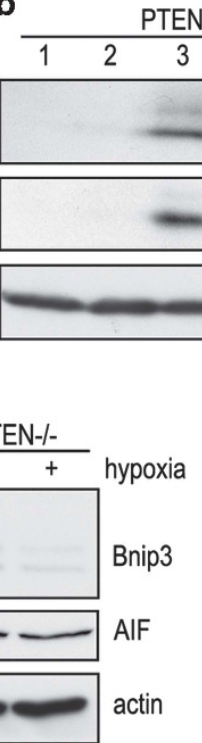

PTEN -/-

\begin{tabular}{llllllll} 
& 4 & 5 & & \multicolumn{5}{c}{ PTEN $-/-$} \\
\cline { 3 - 5 } & 1 & 2 & 3 & 4 & 5
\end{tabular} days

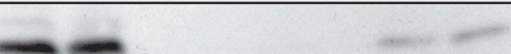

\section{cleaved cas-3}

C

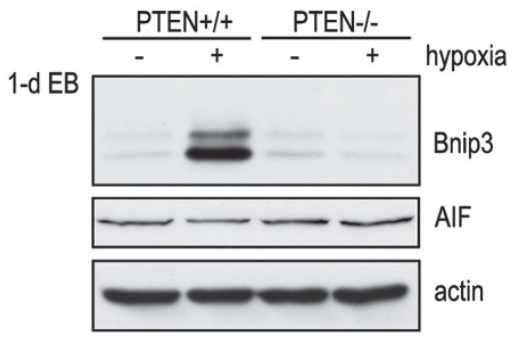

e d $\mathrm{PTEN}+/+\mathrm{PTEN}-/-$

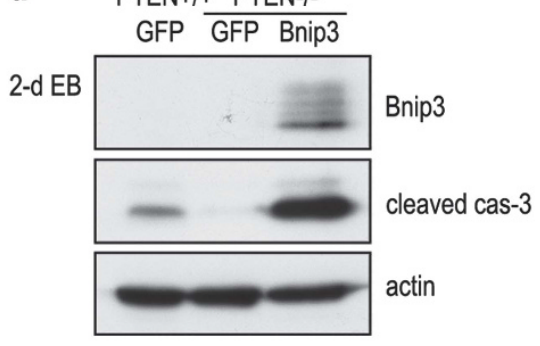

PTEN-/-+Bnip3

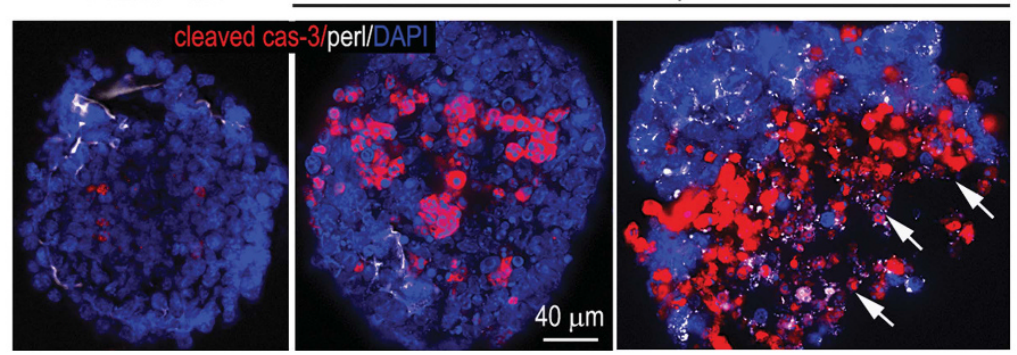

Figure 4 Bnip3 mediates PTEN-dependent apoptosis. (a) Immunoblots show that AlF expression was not significantly altered in 3-day PTEN - / - EBs. (b) Immunoblots demonstrate that Bnip3 and cleaved caspase-3 (cas-3) were markedly upregulated during EB cavitation in PTEN+/+ EBs. Ablation of PTEN attenuated Bnip3 upregulation and inhibited cas-3 activation. (c) One-day EBs were cultured in hypoxia pouches for $16 \mathrm{~h}$ and analyzed by immunoblotting for Bnip3 and AlF. Hypoxia upregulated Bnip3 expression in PTEN+/+ but not PTEN - / - EBs. It had no effect on AlF expression. (d) PTEN+/+ EBs expressing green fluorescent protein (GFP) and PTEN - / - EBs expressing either GFP or Bnip3 were cultured for 2 days. Immunoblots show that overexpression of Bnip3 in PTEN - / - EBs significantly increased cas-3 activation. (e) Immunostaining showed that overexpression of Bnip3 in PTEN - / - EBs significantly increased apoptosis as compared with PTEN - / - GFP EBs

HIF-2a protein is targeted for destruction by prolyl hydroxylation. Among the three prolyl hydroxylase domain-containing proteins (PHDs), PHD2 is ubiquitously expressed and is primarily responsible for HIF- $a$ hydroxylation in both normoxia and hypoxia. ${ }^{25}$ The reduced HIF-2 $a$ in PTEN - / - EBs can also be caused by either increased PHD abundance and/or enhanced enzymatic activity. Immunoblot analysis showed that PHD2 expression was increased nearly threefolds in 4-day PTEN-/ - EBs compared with wild-type controls (Figure 7c). This increased PHD2 expression may also account for HIF-2 $a$ reduction in the mutant EBs. To test this hypothesis, we treated 3-day PTEN-/ - EBs with PHD inhibitor ethyl 3, 4-dihydroxybenzoic acid (DHB) for $24 \mathrm{~h}$. DHB treatment increased HIF-2a, Bnip3 and cleaved caspase-3 (Figure 7d). In line with these findings, HIF-2 $a$ and Bnip3 were elevated in parallel with enhanced caspase-3 activation in 3-day PHD2-/- EBs compared with their wild-type controls (Figure 7e). Taken together, these results suggest that PTEN loss suppresses HIF-2a both transcriptionally and posttranscriptionally, and thus inhibits Bnip3 expression and apoptosis. Furthermore, the posttranscriptional regulation of HIF-2a is mediated at least in part by increased expression of PHD2.

\section{Discussion}

In this study, we show that PTEN is required for both embryonic epithelial polarization and cavitation. Rescue experiments using wild-type PTEN and various mutants suggest that the phosphatase activity of PTEN is dispensable for apoptosis-mediated cavitation. Instead, PTEN loss inhibits hypoxia-induced Bnip3 upregulation via HIF-2a, which in turn induces death of the centrally located cells. These results reveal a novel mechanism whereby PTEN promotes apoptosis and lumen formation during epithelial morphogenesis.

Lumen formation in mammary acini, salivary gland, ureteric buds and the egg cylinder-stage embryo involves cavitation in which the core cells of a solid epithelial primordium are removed by programmed cell death. ${ }^{1,26}$ By using an EB model, which mimics periimplantation development, we 


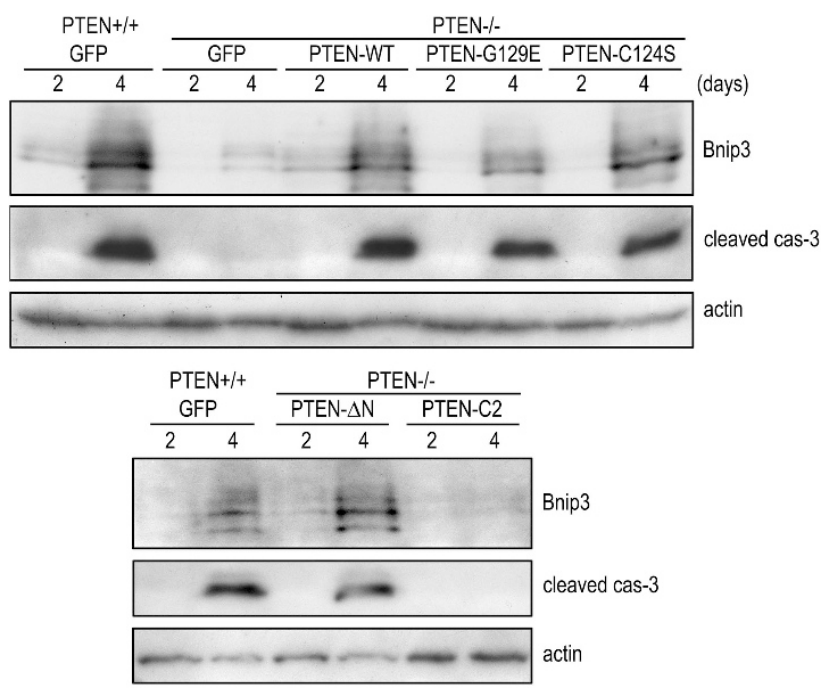

Figure 5 The C-terminal domain of PTEN is required for Bnip3 upregulation and apoptosis. PTEN+/+ green fluorescent protein (GFP) EBs and PTEN - / - EBs expressing GFP, wild-type (WT) PTEN, PTEN-G129E, PTEN-C124S, PTEN- $\Delta \mathrm{N}$ or PTEN-C2 were cultured for 2 and 4 days and analyzed by immunoblotting. The expression of PTEN-WT, PTEN-G129E, PTEN-C124S or PTEN- $\Delta$ N in PTEN - / EBs all rescued Bnip3 induction and caspase-3 (cas-3) activation, whereas the expression of PTEN-C2 had no effect

demonstrate that PTEN-dependent apoptosis and cavitation is not mediated by suppression of the PI3K-Akt pathway. This conclusion is supported by the following findings. ${ }^{1}$ The apoptosis resistance of the core cells but not Akt overactivation could be rescued by reintroduction of phosphatasedeficient PTEN mutants. ${ }^{2}$ Treatment of PTEN - / - EBs with Akt inhibitor attenuated Akt activation but failed to induce central apoptosis. ${ }^{3}$ Overexpression of constitutively active Akt in normal EBs was unable to prevent apoptosis of the core cells. Our mutagenesis analysis further revealed that the $\mathrm{C}$ terminus of PTEN but not the $\mathrm{C} 2$ domain could rescue the central apoptosis. The C2 domain mediates membrane anchorage, while the C-terminal tail contains a PDZ domainbinding motif, which has been shown to interact with PAR3, MAGI2, MAGI3 and NHERF. ${ }^{27}$ The role of the PDZ-binding motif and their binding partners in apoptosis-mediated cavitation warrants further investigation.

PTEN has been shown to reside in various subcellular compartments where it modulates apoptosis through distinct mechanisms. Nuclear PTEN acts together with the tumor suppressor PML to suppress Akt activation, thereby activating FoxO3a-mediated transcription of the proapoptotic protein Bim. ${ }^{28}$ In this study, we also detected PTEN in the nucleus by cytoplasmic nuclear fractionation (Supplementary Figure S4). However, molecular and pharmacological manipulations of Akt and FoxO3a activities did not influence apoptosismediated cavitation. Recently, PTEN at the endoplasmic reticulum (ER) was shown to regulate calcium transfer from ER to mitochondria and thus induce apoptosis in a protein phosphatase-dependent manner. ${ }^{29}$ Moreover, in hippocampal cultures and ischemia-preconditioned hearts, PTEN localized to mitochondria and interacted with the proapoptotic protein Bax to induce cytochrome $c$ release and caspase-3 activation. ${ }^{30,31}$ In contrast to these studies, we did not observe a clear colocalization of PTEN with ER and mitochondria markers in EBs (data not shown). Co-immunoprecipitation showed no interaction between PTEN and Bax. Instead, PTEN was mainly detected in the subplasma membrane region. As the C-terminal PDZ-binding motif of PTEN is likely required for apoptosis induction, we speculate that PTEN at the subplasma membrane compartment may have a role in apoptosis-mediated cavitation.

Recently, we have shown that Bnip3 and AIF cooperate to induce apoptosis and cavitation. ${ }^{19}$ Bnip3 is a hypoxiaresponsive gene and its expression is upregulated in the core cells of early embryos and differentiating EBs. ${ }^{19}$ Although PTEN was also highly expressed in the EB center before cavitation, its protein level was not regulated by acute hypoxia and HIF-1/2a (data not shown). Nevertheless, we show here that Bnip3 upregulation is PTEN-dependent both during EB cavitation and in response to hypoxia. In PTEN-null EBs, Bnip3 upregulation was diminished, but could be restored by transfection with wild-type PTEN or a mutant bearing the $\mathrm{N}$-terminal phosphatase domain deletion. PTEN is likely to act downstream of ROS, at least in part, to mediate Bnip3 elevation as $\mathrm{H}_{2} \mathrm{O}_{2}$ failed to induce Bnip3 expression in PTENnull EBs. Of note, restoration or overexpression of Bnip3 in PTEN - / - EBs rescued apoptosis. In addition, we demonstrated that the protein level of HIF-2a, which responds to chronic hypoxia, ${ }^{32}$ was reduced in PTEN-/ - EBs. Forced expression of constitutively active mutant HIF-2a PPN increased Bnip3 and caspase-3 activation. These novel results support an idea that PTEN induces apoptosis through HIF-2 $a$ and Bnip3 in a phosphatase-independent manner.

How PTEN regulates HIF-2a remains unclear. We observed that HIF-2a was reduced by $\sim 50 \%$ at the mRNA level, suggesting that PTEN controls HIF-2a transcription. In addition, PHD2, the main HIF prolyl hydroxylase expressed in EB, was markedly increased after PTEN ablation. Pharmacological inhibition of PHD activity stabilized HIF-2a, and increased Bnip3 expression and caspase-3 activation. These results indicate PHD2 at least in part contributes to increased HIF-2a protein levels. PTEN can enter the nucleus in response to oxidative stress and following sumoylation. ${ }^{33,34}$ Nuclear PTEN binds to p53 and regulates the expression of hypoxiaresponsive genes and apoptosis. ${ }^{33,35}$ In the present study, PTEN is also detected in the nucleus and acts downstream of ROS to induce Bnip3 expression. It would be interesting to know whether ROS induces PTEN nuclear translocation in EBs. In addition, the $C$ terminus of PTEN has been shown to bind to the nucleolar 58-kDa microspherule protein (MSP58), which forms a complex with the hypoxia-inducible transcription factor DEC1 (differentiated embryo-chondrocyte expressed gene 1) implicated in the pVHL-HIF pathway under chronic hypoxia. ${ }^{36-38}$ It is worth investigating whether these potential binding partners interact with PTEN in EBs and whether they are involved in PHD2 and HIF-2a regulation.

\section{Materials and Methods}

Culturing of ES cell and embryoid bodies. The ES cell lines used for this study were wild-type CJ7, G4 and R1 ES cells, PTEN - / - and PHD2 - / ES cells. ${ }^{3,39-41}$ All the ES cells were cultured on mitomycin C-treated STO cells. EB differentiation was initiated from ES cell aggregates cultured in non-adherent dishes as described. ${ }^{42}$ 
a

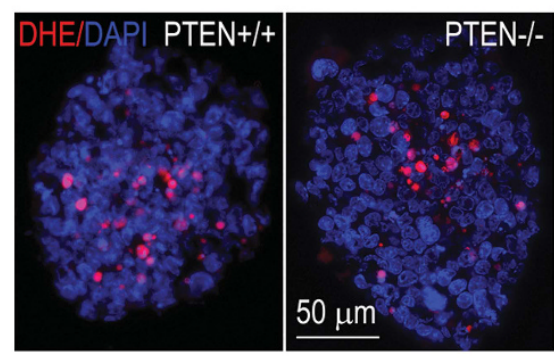

c

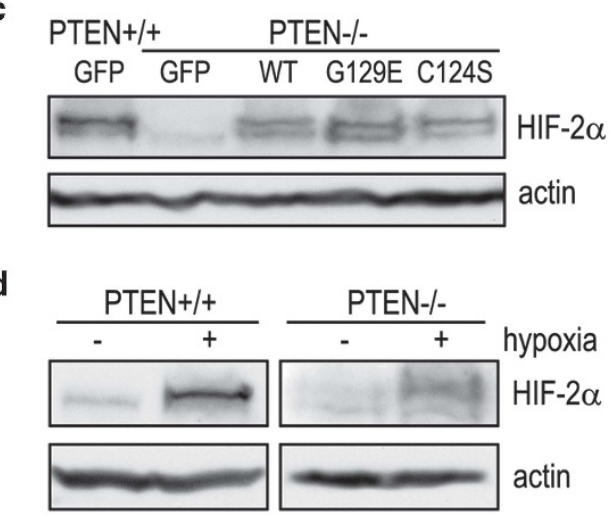

b

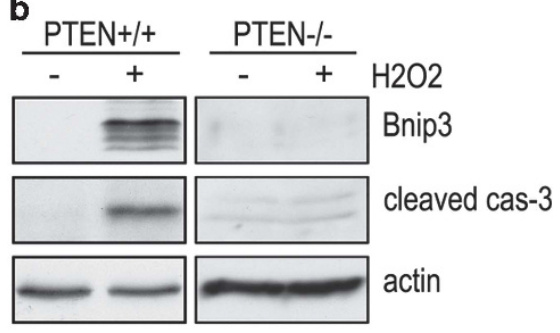

e

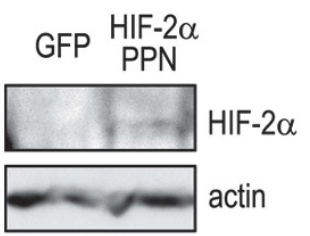

f HIF-2 $\alpha$ GFP PPN

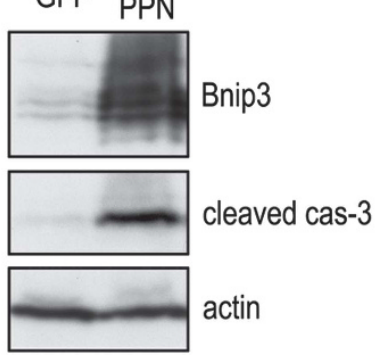

Figure 6 HIF-2 $\alpha$ mediates PTEN-dependent Bnip3 elevation and apoptosis. (a) Three-day PTEN+/+ and PTEN - / - EBs were stained with the fluorescent indicator DHE, which emits red fluorescence after being oxidized by ROS. DHE fluorescence was mainly detected in the core cells in PTEN+/+ EBs and was not significantly changed in the absence of PTEN. (b) One-day EBs were incubated with or without $0.1 \mathrm{mM} \mathrm{H}_{2} \mathrm{O}_{2}$ for $24 \mathrm{~h}$ and harvested for immunoblot analysis. $\mathrm{H}_{2} \mathrm{O}_{2}$ treatment increased Bnip3 and cleaved caspase-3 (cas-3) in PTEN+/+ EBs but not in PTEN - / - EBs. (c) PTEN+/+ GFP EBs and PTEN - / - EBs expressing GFP, wild-type (WT) PTEN, PTEN-G129E or PTENC124S were cultured for 3 days. Immunoblots show that HIF-2 $\alpha$ was markedly decreased in PTEN - / - EBs expressing GFP compared with PTEN+/+ GFP EBs. Reconstitution of PTEN - / - EBs with PTEN-WT, PTEN-G129E or PTEN-C124S all increased HIF-2 $\alpha$ levels. (d) Immunoblots show that hypoxia-induced HIF-2 $\alpha$ elevation was attenuated in PTEN - / - EBs. (e and f) PTEN - / - EBs expressing GFP or oxygen-stable HIF-2 $\alpha$ PPN were cultured for 3 days and analyzed by immunoblotting. Stable expression of HIF-2 $\alpha$ PPN increased Bnip3 expression and cas-3 activation. Actin serves as a loading control

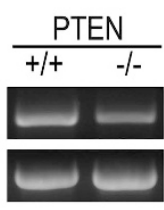

$H I F-2 \alpha$ $18 \mathrm{~S}$

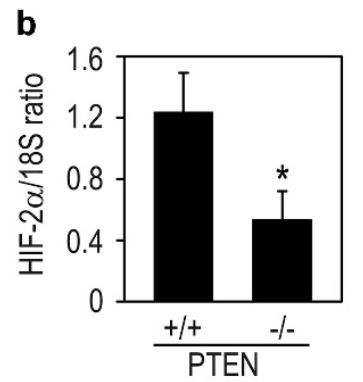

C

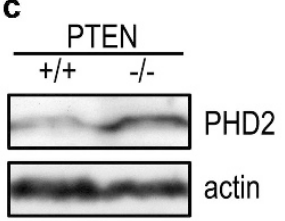

d

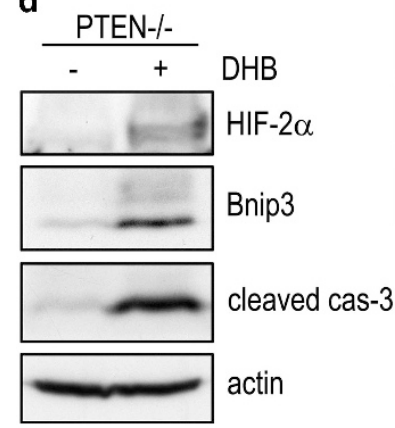

e
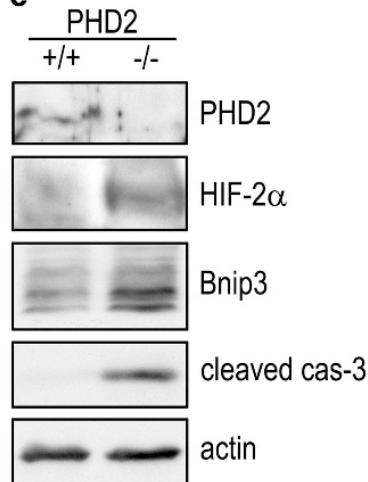

Figure 7 PTEN regulates HIF-2 $\alpha$ expression at both the transcriptional and posttranscriptional levels. (a) RT-PCR analysis showed that the HIF-2 $\alpha$ mRNA was reduced in 4-day PTEN - / - EBs. (b) Ethidium bromide-stained gels were quantitated by densitometry and the ratio of HIF-2 $\alpha$ to $18 \mathrm{~S}$ was plotted $(n=3)$. $P<0.05$ versus PTEN+/+. (c) Immunoblots show that PHD2 expression was increased in 4-day PTEN - / - EBs. (d) Three-day PTEN - / - EBs were untreated or treated with the PHD prolyl hydroxylase inhibitor DHB $(50 \mu \mathrm{M})$ for $24 \mathrm{~h}$. Immunoblots show that DHB treatment increased levels of HIF-2 $\alpha$, Bnip3 and cleaved caspase-3 (cas-3). (e) PHD+/+ and PHD - / - EBs were cultured for 4 days and subjected to immunoblot analysis. PHD2 ablation increased HIF-2 $\alpha$, Bnip3 and cleaved cas-3. Actin serves as a loading control 
Abs and cDNA constructs. Rabbit polyclonal (pAb) or monoclonal (mAb) Abs to cleaved caspase-3, pAkt (Ser473), pAkt (Thr308), Akt, pAkt substrate, PTEN, Bnip3, FoxO3a and fibrillarin were from Cell Signaling (Danvers, MA, USA). Rabbit anti-PTEN pAb was from Cayman Chemical Company (Ann Arbor, MI, USA). Rabbit anti-Bak NT pAb (cat. no. 06-536), rat anti-laminin $\gamma 1$ chain $m A b$ and mouse anti-PTEN and GAPDH mAb were from EMD Millipore (Billerica, MA, USA). Rabbit anti-AIF pAb was from BD Biosciences (San Jose, CA, USA). Rat anti-perlecan mAb was from Santa Cruz Biotechnology (Santa Cruz, CA, USA). Rabbit anti-actin $\mathrm{pAb}$ and mouse anti-Flag-tag and $\beta$-tubulin mAb were from Sigma (St. Louis, MO, USA). Rabbit anti-HIF-2 $\alpha$ pAb was from Novus Biologicals (Littleton, CO, USA). Rabbit anti-PHD2 pAb was from Aviva Systems Biology (San Diego, CA, USA). Mouse anti-complex V (ATP synthase subunit $\beta$ ) was from Life Technologies (Grand Island, NY, USA). Mouse mAb to active Bax (clone 6A7, cat. co. 633802) was from BioLegend (San Diego, CA, USA). Rabbit anti-MUPP1 pAb was provided by Dr. Makoto Adachi of Kyoto University (Kyoto, Japan). Cy3-, Cy5- and horseradish peroxidase-conjugated secondary Abs were from Jackson ImmunoResearch (West Grove, PA, USA). Alexa 488-conjugated secondary Abs were from Molecular Probes (Eugene, OR, USA).

The cDNAs for human wild-type PTEN, the phosphatase-deficient mutants G129E and C124S, human wild-type FoxO3a, the DNA-binding mutant FoxO3a H212R and the triple Akt phosphorylation mutant AAA (T32A, S253A, S315A) were obtained from Addgene (Cambridge, MA, USA ${ }^{43,44}$ and subcloned to PCXN2-IRESGFP. PTEN $\Delta N$ (aa175-403) and C2 (aa175-353) were generated by PCR and cloned to pCXN2-IRES-GFP with an N-terminal Flag tag. The pcDNA3-myr-HA-Akt1 vector, encoding and a myristoylated, constitutively active Akt1 and pBabe-puro HIF-2 $\alpha$ PPN, were obtained from Addgene. ${ }^{44,45}$ The construction of the Bnip3 expression vector was described previously. ${ }^{19}$ All constructs were confirmed by DNA sequencing.

Stable transfection of ES cells. For stable reconstitution of PTEN - / ES cells with wild-type and mutant PTEN, ES cells were transfected with the corresponding vectors using Lipofectamine 2000 reagent (Invitrogen, Grand Island, NY, USA). Stable ES cell clones were selected based on GFP fluorescence and expanded on STO feeder cells. For overexpression of Bnip3, PTEN - / - ES cells were transfected with pCXN2-Bnip3 using Lipofectamine 2000 reagent and also selected for GFP-positive clones. Ectopic expression of HIF-2 $\alpha$ PPN in PTEN - / ES cells was achieved by transfection with Lipofectamine 2000 and stable clones were selected with $1 \mu \mathrm{g} / \mathrm{ml}$ puromycin. Antibiotic-resistant colonies were expanded, and grown in the medium containing $1 \mu \mathrm{g} / \mathrm{ml}$ puromycin. The generation of ES cell clones expressing constitutively active Akt1 was described previously. ${ }^{18}$

Immunofluorescence. EBs were fixed with $3 \%$ paraformaldehyde, embedded in OCT compound and sectioned on a Leica cryostat. EB processing and immunostaining were carried out as described. ${ }^{42}$ Slides were examined with a Nikon inverted fluorescence microscope (Eclipse TE2000, Melville, NY, USA), and digital images were acquired with an Orca-03 cooled charge-coupled device camera (Hamamatsu, Hamamatsu City, Japan) controlled by the IP Lab software (Scanalytics, Milwaukee, WI, USA).

Immunoprecipitation and immunoblotting. EBs were collected by settling by gravity, washed once in phosphate-buffered saline and lysed in radioimmunoprecipitation assay buffer $(50 \mathrm{mM}$ Tris, pH 7.4, $150 \mathrm{mM} \mathrm{NaCl}, 1 \mathrm{mM}$ EDTA, $1 \%$ NP- $40,0.25 \%$ sodium deoxycholate) containing protease and phosphatase inhibitor cocktails (Sigma). For immunoblot analysis of HIF-2 $\alpha$ and PHD2, EBs were lysed in SDS lysis buffer containing $10 \mathrm{mM}$ Tris (pH 7.4), 1\% SDS and protease and phosphatase inhibitors. For immunoblot analysis of active Bak/Bax and oligomer formation, EBs were lysed in CHAPS buffer as described previously. ${ }^{46}$ Immunoprecipitation and immunoblotting were performed as described previously. ${ }^{16}$

RT-PCR. Total RNA was isolated with the TRIzol reagent and reverse transcribed to cDNA using Superscript III reverse transcriptase (RT) (Invitrogen). PCR primers used were $5^{\prime}$-TGGCTCTGGAGTACATCGTG-3' (forward) and 5'-CATGTCACGCAT CTTCCATC-3' (reverse) for mouse HIF-2 $\alpha$ and 5'-GACACCGCCAAATTTAACTG-3' (forward) and 5'-TTCCGCCACTGAACATTG-3' (reverse) for mouse PTEN. 18S RNA was used as a loading control as described previously. ${ }^{47}$

Statistical analysis. Statistical analysis was performed using SigmaStat software (Systat Software, San Jose, CA, USA). Statistical significance was evaluated by one-way analysis of variance and Student's t-test. A $P$-value $<0.05$ was considered significant. Error bars on all figures are represented as a standard deviation of the mean. Blots shown are representative of two to four independent repeats.

Acknowledgements. This study was supported by a grant (R01GM081674) to SL from the National Institutes of Health.

1. Coucouvanis E, Martin GR. Signals for death and survival: a two-step mechanism for cavitation in the vertebrate embryo. Cell 1995; 83: 279-287.

2. Salmena L, Carracedo A, Pandolfi PP. Tenets of PTEN tumor suppression. Cell 2008; 133 : 403-414.

3. Di Cristofano A, Pesce B, Cordon-Cardo C, Pandolfi PP. Pten is essential for embryonic development and tumour suppression. Nat Genet 1998; 19: 348-355.

4. Podsypanina K, Ellenson LH, Nemes A, Gu J, Tamura M, Yamada KM et al. Mutation of Pten/ Mmac1 in mice causes neoplasia in multiple organ systems. Proc Natl Acad Sci USA 1999; 96: $1563-1568$

5. Suzuki A, de la Pompa JL, Stambolic V, Elia AJ, Sasaki T, del Barco Barrantes I et al. High cancer susceptibility and embryonic lethality associated with mutation of the PTEN tumor suppressor gene in mice. Curr Biol 1998; 8: 1169-1178.

6. Bardet PL, Guirao B, Paoletti C, Serman F, Leopold V, Bosveld F et al. PTEN controls junction lengthening and stability during cell rearrangement in epithelial tissue. Dev Cell 2013; 25: 534-546

7. Pinal N, Goberdhan DC, Collinson L, Fujita Y, Cox IM, Wilson C et al. Regulated and polarized Ptdlns $(3,4,5) \mathrm{P} 3$ accumulation is essential for apical membrane morphogenesis in photoreceptor epithelial cells. Curr Biol 2006; 16: 140-149.

8. Martin-Belmonte F, Gassama A, Datta A, Yu W, Rescher U, Gerke V et al. PTEN-mediated apical segregation of phosphoinositides controls epithelial morphogenesis through $\mathrm{Cdc} 42$. Cell 2007; 128: 383-397.

9. Berglund FM, Weerasinghe NR, Davidson L, Lim JC, Eickholt BJ, Leslie NR. Disruption of epithelial architecture caused by loss of PTEN or by oncogenic mutant p110alpha/PIK3CA but not by HER2 or mutant AKT1. Oncogene 2013; 32: 4417-4426.

10. Maehama T, Dixon JE. The tumor suppressor, PTEN/MMAC1, dephosphorylates the lipid second messenger, phosphatidylinositol 3,4,5-trisphosphate. J Biol Chem 1998; 273: 13375-13378.

11. Tamura M, Gu J, Matsumoto K, Aota S, Parsons R, Yamada KM. Inhibition of cell migration, spreading, and focal adhesions by tumor suppressor PTEN. Science 1998; 280: 1614-1617.

12. Gu J, Tamura M, Pankov R, Danen EH, Takino T, Matsumoto $K$ et al. Shc and FAK differentially regulate cell motility and directionality modulated by PTEN. J Cell Biol 1999; 146: 389-403.

13. Raftopoulou M, Etienne-Manneville S, Self A, Nicholls S, Hall A. Regulation of cell migration by the C2 domain of the tumor suppressor PTEN. Science 2004; 303: 1179-1181.

14. Shen WH, Balajee AS, Wang J, Wu H, Eng C, Pandolfi PP et al. Essential role for nuclear PTEN in maintaining chromosomal integrity. Cell 2007; 128: 157-170.

15. Song MS, Carracedo A, Salmena L, Song SJ, Egia A, Malumbres $M$ et al. Nuclear PTEN regulates the APC-CDH1 tumor-suppressive complex in a phosphataseindependent manner. Cell 2011; 144: 187-199.

16. Li S, Harrison D, Carbonetto S, Fassler R, Smyth N, Edgar D et al. Matrix assembly, regulation, and survival functions of laminin and its receptors in embryonic stem cell differentiation. J Cell Biol 2002; 157: 1279-1290.

17. Li S, Edgar D, Fassler R, Wadsworth W, Yurchenco PD. The role of laminin in embryonic cell polarization and tissue organization. Dev Cell 2003; 4: 613-624.

18. He X, Liu J, Qi Y, Brakebusch C, Chrostek-Grashoff A, Edgar D et al. Rac1 is essential for basement membrane-dependent epiblast survival. Mol Cell Biol 2010; 30: 3569-3581.

19. Qi Y, Tian X, Liu J, Han Y, Graham AM, Simon MC et al. Bnip3 and AIF cooperate to induce apoptosis and cavitation during epithelial morphogenesis. J Cell Biol 2012; 198: 103-114.

20. Myers MP, Pass I, Batty IH, Van der Kaay J, Stolarov JP, Hemmings BA et al. The lipid phosphatase activity of PTEN is critical for its tumor supressor function. Proc Natl Acad Sci USA 1998; 95: 13513-13518.

21. Maier D, Jones G, Li X, Schonthal AH, Gratzl O, Van Meir EG et al. The PTEN lipid phosphatase domain is not required to inhibit invasion of glioma cells. Cancer Res 1999; 59 : 5479-5482.

22. Hagenbuchner J, Ausserlechner MJ. Mitochondria and FOXO3: breath or die. Front Physiol 2013; 4: 147

23. Tzivion $\mathrm{G}$, Dobson M, Ramakrishnan G. FoxO transcription factors; regulation by AKT and 14-3-3 proteins. Biochim Biophys Acta 2011; 1813: 1938-1945.

24. Kubli DA, Ycaza JE, Gustafsson AB. Bnip3 mediates mitochondrial dysfunction and cell death through Bax and Bak. Biochem J 2007; 405: 407-415.

25. Kaelin Jr WG, Ratcliffe PJ. Oxygen sensing by metazoans: the central role of the HIF hydroxylase pathway. Mol Cell 2008; 30: 393-402.

26. Debnath J, Mills KR, Collins NL, Reginato MJ, Muthuswamy SK, Brugge JS. The role of apoptosis in creating and maintaining luminal space within normal and oncogene-expressing mammary acini. Cell 2002; 111: 29-40.

27. Feng W, Wu H, Chan LN, Zhang M. Par-3-mediated junctional localization of the lipid phosphatase PTEN is required for cell polarity establishment. J Biol Chem 2008; 283 . 23440-23449. 
38. Choi SM, Cho HJ, Cho H, Kim KH, Kim JB, Park H. Stra13/DEC1 and DEC2 inhibit sterol regulatory element binding protein-1c in a hypoxia-inducible factor-dependent mechanism. Nucleic Acids Res 2008; 36: 6372-6385.

39. Wu X, Li S, Chrostek-Grashoff A, Czuchra A, Meyer H, Yurchenco PD et al. Cdc42 is crucial for the establishment of epithelial polarity during early mammalian development. Dev Dyn 2007; 236: 2767-2778.

40. Smyth N, Vatansever HS, Murray P, Meyer M, Frie C, Paulsson M et al. Absence of basement membranes after targeting the LAMC1 gene results in embryonic lethality due to failure of endoderm differentiation. J Cell Biol 1999; 144: 151-160.

41. Fassler R, Pfaff M, Murphy J, Noegel AA, Johansson S, Timpl R et al. Lack of beta 1 integrin gene in embryonic stem cells affects morphology, adhesion, and migration but not integration into the inner cell mass of blastocysts. J Cell Biol 1995; 128: 979-988.

42. Li S, Yurchenco PD. Matrix assembly, cell polarization, and cell survival: analysis of peri-implantation development with cultured embryonic stem cells. Methods Mol Biol 2006; 329: $113-125$.

43. Vazquez F, Grossman SR, Takahashi Y, Rokas MV, Nakamura N, Sellers WR Phosphorylation of the PTEN tail acts as an inhibitory switch by preventing its recruitment into a protein complex. J Biol Chem 2001; 276: 48627-48630.

44. Ramaswamy S, Nakamura N, Vazquez F, Batt DB, Perera S, Roberts TM et al. Regulation of G1 progression by the PTEN tumor suppressor protein is linked to inhibition of the phosphatidylinositol 3-kinase/Akt pathway. Proc Natl Acad Sci USA 1999; 96: 2110-2115.

45. Yan Q, Bartz S, Mao M, Li L, Kaelin WG Jr. The hypoxia-inducible factor 2alpha N-terminal and $\mathrm{C}$-terminal transactivation domains cooperate to promote renal tumorigenesis in vivo. Mol Cell Biol 2007; 27: 2092-2102.

46. Upreti M, Chu R, Galitovskaya E, Smart SK, Chambers TC. Key role for Bak activation and Bak-Bax interaction in the apoptotic response to vinblastine. Mol Cancer Ther 2008; 7 2224-2232.

47. Liu J, He X, Qi Y, Tian X, Monkley SJ, Critchley DR et al. Talin1 regulates integrin turnover to promote embryonic epithelial morphogenesis. Mol Cell Biol 2011; 31: 3366-3377.

Supplementary Information accompanies this paper on Cell Death and Differentiation website (http://www.nature.com/cdd) 